\title{
Improving clinical handover: Development of a web-based intensive care unit consultation system with structured reply generation
}

\author{
Jasperine Ka-yee Ho
}

New Territories West Cluster, Hospital Authority of Hong Kong

\begin{abstract}
Clinical handover is a process of transferring a patient's clinical information from one team to another. Poor quality of handover of critically ill patients associates with misunderstanding, delay in treatment, and adverse outcomes. Therefore, a high quality and standardized information flow process and documentation are definitely crucial. We have developed a web-based platform for generating printed ICU consultation reply and data collection. Baseline measurement was performed by randomly identified 50 case records among 220 ICU consultations in March 2015 , before the implementation of the system. We found that there were only $16 \%$ of cases had printed ICU consultation reply, $42 \%$ had documented patient's premorbid activity of daily living, $30 \%$ had recorded patient's mobility, $74 \%$ had written down patient's past medical history, $72 \%$ had clearly stated the ICU admission decision, $18 \%$ had provided the reasons for rejecting ICU admission, and $32 \%$ had remarked the mechanism for contacting ICU senior to review the admission. After implementation of the ICU consultation reply platform, the percentage of using printed consultation reply has increased to more than $90 \%$, documentation of patient's morbidity and mobility have raised to over $80 \%$ and patient's past medical history has been recorded in over $95 \%$ of cases. Standardised ICU admission decision tree has been designed to assist ICU doctor to clearly state the ICU admission decision and the reasons behind. Appeal mechanism for doctors in general ward to contact ICU seniors for case discussion is included in every consultation reply. In short, ICU consultation platform improves the quality of ICU consultation reply and enhances communication among ICU doctors and general ward staff. It also serves as a big data pool for audit cycles which in turns direct ICU consultation service improvement.
\end{abstract}

\section{Problem}

Tuen Mun Hospital (TMH) is one of the busiest acute hospitals in Hong Kong. Intensivists working at TMH are not only taking care of critically ill patients in the intensive care unit (ICU) but also responsible for emergency intubation, resuscitation, attending trauma and arrest call, and triage for ICU admission in general wards and emergency department. On average, we receive 7.5 emergency consultations per day (916 emergency consultations between July 2015 and October 2015).

When there is a critically ill patient in the ward, an ICU doctor holding a mobile phone "6853" is called for attendance. A brief verbal handover consisting of patient's past health, working diagnosis and management, vital signs, and reasons for ICU consultation is discussed on the phone. The ICU doctor then prioritizes the clinical urgency of each consultation based on the information provided by consulting doctors and assesses patients one by one.

Before the implementation of this electronic platform, ICU doctors wrote clinical assessments and ICU admission decisions on a patient's clinical paper record after resuscitation. Then, the patient's gum label with patient's particulars printed on it was collected. ICU doctors might only be able to return to ICU after assessing several patients. Details of ICU consultations were summarized and patients' gum labels were stuck on a ICU consultation book for clinical handover purposes.

Feedback from clinical departments regarding the quality of ICU consultation reply was received. These included illegible handwriting, unclear ICU admission decisions, and no appeal mechanism for general team doctors to liaise with ICU seniors on ICU admission decision. Apart from the suboptimal quality of the ICU consultation reply to the general team, the documentation on ICU consultation book for handover among ICU teammates were below the expected standard. Therefore, an audit on items considered to be essential to include in ICU consultation reply was conducted.

\section{Background}

The importance of clinical handover between ICU and general ward staff for patients discharging from ICU has been extensively discussed in the literature.[1-4] However, documentation of ICU assessment done for critically ill patients in general wards is rarely studied. The journey of a critically ill patient is complex and hence a multidisciplinary care approach is often required, with consultation becoming the cornerstone of optimising standards of patient care. Clinical handover is an important part of ICU consultation; nevertheless, the majority of published studies focused on the discussion of documentation of ICU discharge. One original study published in 2013 highlighted that a high quality ICU consult should compose of quality of handover, decisiveness, timeliness, professionalism, and team coordination.[3] The General Medical Council (GMC) has clearly stated that a medical record should include relevant clinical findings; decisions made and actions agreed, and who is making the decisions and agreeing the actions.[5] Tips for good record keeping suggested by the Medical Defence Union include legible writing, full details of the patient, and 
the date and time.[6]

Completed in 1989, TMH is an 1,800 bed acute regional hospital, providing a comprehensive range of specialty services in secondary and tertiary care in the New Territories West Cluster (NTWC) of Hospital Authority (HA) in Hong Kong. The average number of hospital admissions is 90,000 per year and the rate of ICU consultation is 8 to 14 per 1,000 admissions. A high quality ICU consultation service is a reasonable expectation from both patients and general teams. The aim of this project is to improve the quality of ICU consultation reply and enhance communication among ICU doctors and general ward staff. Developing a web-based ICU consultation system (ICON) also serves as a big data pool for audit cycles which in turn directs ICU consultation service improvement. The ICON developing team includes ICU doctors, representatives from Quality and Safety Division (Q\&S), and Information Technology (IT) department of TMH.

See supplementary file: ds7305.docx - "ICON steps and ICU decision tree"

\section{Baseline measurement}

The items chosen in the baseline measurement are based on the GMC recommendation on medical records and the Medical Defense Union on good record keeping. In addition, qualitative feedback from ICU team seniors and clinical departments on the expectation of history taking and documentation on the clinical details guiding ICU admission triage decision were included.

In March 2015, there were 220 ICU consultations recorded in the ICU consultation book. A list of hospital numbers with assigned number 1 to 220 was generated and randomization was performed by using excel function. The first 50 cases, around a quarter of the total, were used for the baseline measurement analysis. Patient's hospital records were loaned and data were filled in a form consisting of item 1 to 10 . Collected data were further analyzed and graphs were drawn by excel functions. The compliance of the following domains was retrospectively collected via case record review:

1. ICU consultation reply in printed format: $8 / 50(16 \%)$

2. Clearly document ICU assessment date and time: $49 / 50$ (98\%)

3. Clearly document ICU doctor's name or code: $45 / 50$ (90\%)

4. Clearly state patient's premorbid activity of daily living: $21 / 50(42 \%)$

5. Clearly state patient's mobility: $15 / 50(30 \%)$

6. Clearly state patient's past medical history: $37 / 50$ (74\%)

7. Legible handwriting: $47 / 50$ (94\%)

8. Clearly state the ICU admission decision: $36 / 50$ (72\%)

9. Clearly state the reasons for rejecting ICU admission: $9 / 50$ (18\%)

10. Clearly state the mechanism of contacting ICU senior for discussion: 16/50 (32\%).

The baseline measurement demonstrated there were areas of good practice, particularly regarding the documentation of ICU doctor's name or code, ICU assessment date and time and legibility of handwriting. However, there was a lack of clarity in terms of patient's premorbid status, ICU admission decision, reasons for rejecting ICU admission, and the mechanism for contacting ICU senior to review the admission decision.

\section{Design}

We received negative feedback from clinical departments relating to the documentation of our ICU consultation reply, which created a sense of urgency among ICU team members to improve our communication with clinical teams. After analysing the feedback, we found that almost all could be resolved by developing a tool which would guide our frontline doctor to write a high quality summary and generate a printed out reply sheet.

Literature review on the quality of ICU consultation and predicting model for ICU mortality on ICU admission was conducted.[7-10] Followed by that, we had face to face interviews with frontline and senior clinicians of Department of Medicine and Department of Surgery, accounting for more than $60 \%$ of ICU referrals. ICON was designed based on the conclusion of the interviews and literature review. In the information technology era, transforming paper documentation to electronic platform is an innovative approach to improve medical documentation and clinical handover.

We had collaborated with the Quality and Safety Division (Q\&S) and Information Technology Department (IT) of TMH and designed ICON under the webpage of Department of Anaesthesia and Intensive Care.

Steps for using the ICON after accessing a critically ill patient in general wards by ICU doctor can be found in the attached document (ICON steps and ICU decision tree). The ICU decision tree has been created as part of the project. It consists of the majority of clinical scenarios and is used to facilitate the ICU doctor during the triage process and clearly document the reasons for the ICU admission decision.

Face-to-face handover on critically ill patients always plays an essential role, the ICU doctor would still discuss with the general team doctor and nurse about the management plan and follow-up action besides leaving the ICON generated reply sheet in patient's medical record.

The beauty of this web-based ICU consultation system is that the ICU doctor could approach any computer which linked to the hospital intranet system inside the ward to submit details of the ICU consultation and print out the consultation reply in one. Since we no longer use handwritten clinical notes in ICU for more than eight years, our ICU teammates are used to electronic medical documentation.

We strongly encourage the ICU doctor to use 2D barcode scanner to scan on the patient's gum label when they submit patient's hospital number. A check digit function, which counterchecks the accuracy of hospital number submitted, is included in the system. The ICON uses the time which ICU doctor logs in the system as the 
"ICU consultation date and time". This information is used to capture ICU doctors response time yet we know there could be significant time lag between the ICU doctors arrival time and ICU consultation notes typing time which may only be started after patient resuscitation. Therefore, we allow manual change in the capture time so as to collect the factual data. Definitions on items under SCCM classification, patient's quality of life, physiology, chronic diagnosis, and acute diagnosis are shown on the right hand side of the screen when a small icon next to each title of those terms is clicked. ICU doctors could recall the detailed definitions in case they need a reference.

All ICU doctors in the unit could read submitted cases via the ICON but amendment is generally not allowed except the one who submits the case can make corrections within 24 hours. This function facilitates ICU clinical handover and doctors could trace patient's information and the progress via the hospital intranet system.

\section{Strategy}

PDSA 1

The aim is to enhance the ICU consultation reply and data collection quality. After analyzing the baseline measurements, we have developed a web-based ICU consultation data entry and printed out reply generating tool to achieve the target of providing a high quality ICU consultation assessment, ICU reply, and data collection. ICU doctor inserts patient's demographic data, premorbid and past medical history, history of present illness, ICU admission decision and reasoning via the web-based system immediately after patient's assessment in ward and prints out the electronic filled reply form for filling.

In July 2015, there were 276 ICU consultations submitted via ICON. 5 out of $276(1.81 \%)$ consultation details were incompletely entered. The rate of data completeness got worse in August 2015, 19 out of 197 (9.64\%) of consultations were partially filled. Doctors put down "please refer to clinical notes for details" or just a few sentences summarizing the assessment in the electronic system. Instead of using the system to type and generate the reply form, they used their pens to write on paper in patients' records. Feedback relating to ICU admission decision tree was received. ICU doctors expressed the difficulty in assigning the admission decision as some patients were waiting for urgent investigations which guided plan of management and prognosis when they were assessed. Furthermore, some patients were rejected for ICU admission because they were granted Cardiac Care Unit (CCU) admission for cardiac problems.

PDSA 2

We aim to improve the compliance and quality of ICU consultation data entry and printed out reply. After learning the above, a second cycle of education was conducted on 1 September 2015. Education and emphases on the importance of ICU consultation database, live demonstration on the whole process of data submission, explanation of the meaning of each term were repeated. Since
ICON is also a tool facilitating consultation handover among ICU teammates, the system is accessed twice per day. Besides case discussion, seniors also share opinions on the quality and accuracy of data submission like how to grade SCCM category.

To address the difficulties in assigning appropriate ICU admission decisions, two new options have been added to the decision tree: "Please inform ICU once further management plan has been established" and "The patient will be transferred to CCU for further management." Under the Hawthorne effect and after reiteration of the purpose of the project, the compliance of data entry and usage of printed out ICU consultation reply has increased.

See supplementary file: ds7571.docx - "PDSA cycle 1 and cycle 2"

\section{Post-measurement}

There were 231 and 212 ICU consultations in September 2015 and October 2015 respectively. After implementation of the ICU consultation reply platform, the percentage of using printed consultation reply has increased to more than $90 \%$, documentation of patient's morbidity and mobility has raised to over $80 \%$ and patient's past medical history has been recorded in over $95 \%$ of cases. ICU consultation platform improves the quality of ICU consultation reply and enhances communication among ICU doctors and general ward staff. (See data collected and results graph)

See supplementary file: ds7570.docx - "Data collected and results"

\section{Lessons and limitations}

We encountered difficulty in retrieving data to analyze our ICU consultation performance and resources planning. Seniors also wished to have an electronic platform through which they can glance at cases reviewed by ICU frontline doctors anytime and anywhere within the hospital. For frontline staff, all of us are familiar with and used to communicating through electronic means and are good at typing. All these positive drives decide the success of our project.

ICU is a place for doctors from other specialties to expose critically ill patients and their management. There are new staff rotating into our unit three-monthly, regular introduction and sharing on the concept and terms of the ICU consultation platform is necessary to ensure the compliance and accuracy of data collection. We explained the reasons for developing this ICON platform and demonstrated to them how to submit cases in a step-by-step manner. Furthermore, ICON is used to facilitate ICU consultation case handover during ICU handover round, seniors could screen the compliance, data submission, and correct misconceptions to frontline doctors instantly. Regular audit is going to be conducted half-yearly.

Another limitation is the availability of computer in nursing station in general wards. Doctors, nurses, allied health, and health care assistants read patients' past health and make requests such as 
laboratory tests, imaging tests, meal orders, and interdepartmental consultation via intranet through computers. ICU doctor may need to spend time on queuing. The worst situation is during trauma call in the Emergency Department. There is only one computer in each trauma room. We are exploring the possibility of using electronic device such as tablet or mobile phone which could link to the hospital computer system so that our doctor could do documentation at bedside. We may further enhance the system by adding the apps for photo taking and for diagram drawing to allow better documentation.

\section{Conclusion}

In the literature, a majority of the studies related to ICU clinical handover focus on ICU discharge handover. On the contrary, this work points out the importance of pre-ICU admission documentation and ICU consultation reply. By designing a userfriendly web-based electronic ICU consultation reply and database collection tool with hospital IT and Q\&S division, the whole process of critically ill patient's assessment in general wards is modified. Frontline ICU doctors type ICU consultation reply via an electronic platform immediately after resuscitation, this helps to reduce recall information bias. A structured ICU consultation reply form involves exact consultation date and time, name and code of assessing doctor, patient's assessment record, clear ICU admission decision and the reasoning behind it, and appeal mechanism is printed for general ward staff. General ward doctors and nurses can follow the suggestions easily and they know who to approach if further discussion is considered appropriate. A clear written communication between ICU and general team reduces misunderstanding and reduces the time for clarification and potentially reduces near misses and medical incidents. Further study shall be considered to look into these impacts. The platform also serves as a handover tool during ICU handover rounds. Regular update and teaching to ICU core team members and rotating staff is arranged to ensure sustainability as well as the compliance and accuracy of data submission. Last but not least, the ICU consultation database serves as a big data pool for audit cycles which direct ICU consultation service improvement. The ICON could be replicated to other ICUs which are still using a handwritten reply form.

\section{References}

1 Nelleke VS, Gijs H, Johannes GH, et al. Improving clinical handover between intensive care unit and general ward professionals at intensive care unit discharge. Intens Care Med (2015) 41:589-604.

2 Stephanie M, Saeid E, Marjan A. Improved communication in postICU care by improving writing of ICU discharge letters: a longitudinal before-after study. MJ Qual Saf 2011;20:967e973.

3 Jennifer P, Stevens AC, Mara AS, et al. Elements of a HighQuality Inpatient Consultation in the Intensive Care Unit. A Qualitative Study. Annals ATS Volume 10 Number 3 June 2013.
Process in an Intensive Care Unit (ICU): Barriers and Quality Improvement Strategy. Int J Qual Health Care 2015;27(2):99-104

5 General Medical Council. The duties of a doctor registered with the General Medical Council 2013.

6 Medical Defense Union. MDU factsheet for consultants. MDU, 2010.

7 Guidelines for intensive care unit admission, discharge, and triage. Task Force of the American College of Critical Care Medicine, Society of Critical Care Medicine. Crit Care Med 1999 Mar;27(3):633-8.

8 Jean-Louis V, Rui M. Clinical review: Scoring systems in the critically ill. Crit Care 2010;14:207.

9 Brian N, Wayne C, Maureen S, et al. Mortality probability models (MPMO-III) for specialised patient populations. Chest 2005;128 (4_MeetingAbstracts):349S.

10 Higgins TL, Teres D, Copes WS, et al. Assessing contemporary intensive care unit outcome: an updated Mortality Probability Admission Model (MPM0-III). Crit Care Med 2007 Mar;35(3):827-35.

\section{Declaration of interests}

We have read and understood BMJ policy on declaration of interests and declare that we have no competing interests.

\section{Acknowledgements}

The Jockey Club School of Public Health and Primary Care, The Chinese University of Hong Kong, Professor Fung Hong, Dr Tang Kam Shing, Dr Lam Kwok Kei, Dr Chan King Chung, Dr Kwan Wai Man, Dr Chan Yan Fat, Dr Ho Chun Ming, Dr Wong Oi Fung, Dr Yip Hing Wah, Dr Fong Man Chi, Dr Yim Tze Kin, Dr Chan Kai Wing, Dr Cham Yin Kwan, Dr Chan Sze Man, Dr Chan Cheuk Yan, Dr Tong Man Chun, Dr Ling Kin Ho, Dr Mak Kwok Shing, Dr Lam Ki Wai, Dr Wu Ping, Dr Ng Chi Wai, Mr Wallace Cheung, Mr Law Chi Ho, Ms Crystal Leung, Mr Jaden Lam, Ms Vivian Kwong.

\section{Ethical approval}

According to the policy activities that constitute research at the Tuen Mun Hospital, this work met criteria for operational improvement activities exempt from ethics review. 